\title{
Time in probabilistic causation: Direct vs. indirect uses of lexical causative verbs ${ }^{1}$
} Fabienne MARTIN — Universität Stuttgart

\begin{abstract}
It is traditionally assumed that lexical causative verbs (e.g. kill) express direct causation only, while periphrastic (bi-clausal) causatives (e.g. cause to die) may also express indirect causation. In favour of this constraint, Fodor famously observed that the (change of) state introduced by lexical causative verbs is not accessible for separate adverbial modification by temporal (or manner) adverbials. In this paper, I present old and new arguments against the direct causation constraint under the definitions of directness of Fodor and Wolff. I then propose a new definition of directness in terms of ab-initio causal sufficiency framed in Kvart's probabilistic account of singular causation. I argue that directness so redefined is an implicature rather than an entailment of lexical causative verbs, which enables me to account for old and new data. Furthermore, I account for why the constraint on separate modification by temporal adverbials can be relaxed with eventuality-denoting subjects.
\end{abstract}

Keywords: lexical causative verbs, direct vs. indirect causation, causal sufficiency, probabilistic theories of causation, semantics/pragmatics interface.

\section{The direct causation constraint}

It is traditionally assumed that lexical causative verbs (e.g. kill) express direct causation only, while periphrastic (bi-clausal) causatives (e.g. cause to die) may also express indirect causation. This constraint associated to lexical causative verbs, which I will call the 'direct causation constraint', has been defended under various forms by Ruwet (1972), Levin and Rappaport Hovav (1999) and Wolff (2003) among others. In favour of this constraint, Fodor (1970) famously observed that the (change of) state introduced by lexical causative verbs is not accessible for separate adverbial modification by temporal (or manner) adverbials, see (1).

(1) a. *Floyd melted the glass on Sunday by heating it on Saturday.

b. Floyd caused the glass to melt on Sunday by heating it on Saturday.

According to Fodor, this syntactic constraint ultimately reflects a semantic restriction on the type of causation events that lexical causatives describe; namely, the causal relation they encode may only have temporally adjacent events as relata. ${ }^{2}$ The same view is endorsed by Katz (1970), who

\footnotetext{
${ }^{1}$ I am very grateful to Zsófia Gyarmathy and Christopher Piñón for extensive feedback, as well as to Jean-Pierre Koenig, Uli Sauerland, Florian Schäfer, Giorgos Spathas, the reviewers and audience of Sinn und Bedeutung 22 and the audience of the Linguistic Perspectives on Causation Workshop (Jerusalem, June 2017) for their very helpful comments. I also want to thank Katharina Fezer and Margaret Grant for the data collection in English and French, as well as Zsófia Gyarmathy and the editors of the Proceedings for proofreading and commenting on a former version of this paper. I am responsible for all remaining mistakes. This work is part of project B5 of the SFB 732 supported by the DFG and hosted by the University of Stuttgart.

${ }^{2}$ As he put it, 'One can cause [something to melt] by doing something at a time which is distinct from the [melting event]. But if you melt something, then you melt it when it melts.' (Fodor 1970: 433)
} 
argues that the sentence in (2b) is false in what I will call the 'Wild West story' (2a), to which I will come back on several occasions below.

(2) a. The sheriff's six-shooter is faultily repaired by the gunsmith. As a result, two days later, the sheriff's gun jams during a gunfight with the terrible Fred in a sordid pub, and the sheriff is shot to death.

b. The gunsmith killed the sheriff.

Fodor and Katz understand directness as requiring temporal adjacency between the cause and the effect, so that no third event is allowed to intervene. But an alternative definition has been provided by Wolff (2003). In section 2, I present old and new arguments against the direct causation constraint under both definitions of directness. I then offer a new definition of directness in section 3, framed in Kvart's (2001) probabilistic account of singular causation, and argue that directness so redefined is an implicature rather than an entailment of lexical causative verbs, what will enable me to account for the data presented in section 2. Finally, in section 4, I explain why separate modification by temporal adverbials is possible in some cases.

\section{Against the direct causation constraint}

Neeleman and Van de Koot (2012) offer a rich list of examples where the causing event is separated from its result by intermediate events, see e.g. (3). Note that most of these examples involve eventor state-denoting subjects. ${ }^{3}$

(3) a. NHS supplies chaos killed my brother.

b. The gunsmith's negligence killed the sheriff.

c. Opening bus lanes to motorcycles will redden the streets of London with cyclists' blood.

In the tradition of discourse theory, Danlos (2001) relatedly observed that lexical causative verbs can be used when indirect causation is involved, as long as the restriction on separate adverbial modification observed by Fodor is respected, see her example (4).

(4) Fred killed Masha. He fired a shot at her on Sunday. She had an hemmorhage. She died on Monday.

Rappaport Hovav and Levin (2001: 783) also mention in passing that subevents of lexical causatives need not be temporally adjacent, observing that in their example (5), the act of putting arsenic in the coffee does not extend to the point where the drinker dies.

\footnotetext{
${ }^{3}$ As already observed by R. Truswell in a p.c. reported in Neeleman and Van de Koot (2012: fn. 9). Neeleman and Van de Koot (2012) claim that this is not a condition for licensing indirect causal chains with lexical causatives, which I agree with, but it often facilitates this reading, what will be accounted for in section 3.
} 
(5) The widow murdered her guest by putting arsenic in his coffee.

But in fact, even the restriction on separate adverbial modification can be relaxed in some contexts. ${ }^{4}$ For instance, my English and French informants all converge in the view that in presence of an event-denoting subject, separate adverbial modification is possible, see the English contrast $(6 \mathrm{a} / \mathrm{b})$, as well as the French contrast (7).

(6) a. Fred accidentally shot his dog on December 23! \#He eventually killed him on December 25.

b. Fred accidentally shot his dog on December 23! This gunshot eventually killed him on December 25.

(7) M. $\operatorname{Roy}_{i}$ a secoué $_{j}$ son bébé de 3 mois. Résultat, 69 jours après, \#il ${ }_{i} /{ }^{O K}$ ça $_{j}$ Mr Roy has shaken his baby of 3 months as a result 69 days later he/ this a fini par le tuer. has finished by him kill

'Mr Roy shook his 3 months old baby. As a result, 69 days later \#he/ ${ }^{O K}$ this eventually killed him.'

In both (6) and (7), the version with an individual-denoting subject is generally judged not acceptable. ${ }^{5}$ On the other hand, however, the version with an event-denoting subject is acceptable (under the relevant reading where the event denoted by the subject of the second clause refers back to the event introduced in the first clause, and thus takes place days before the time specified by the temporal adverbial in the second clause) ${ }^{6}$

In (7), the presence of the verb finir par $P$, translatable by end up $P$-ing or eventually/ultimately $P$, is not innocent. This verb seems to facilitate the indirect reading of lexical causative verbs, as does the adverbial eventually in (6b). To illustrate the point more explicitly, I borrow from Lauer and Nadathur (2017) one of their scenarios repeated under (8). ${ }^{7}$ In this scenario, (8a) is inappropriate, but $(8 \mathrm{~b} / \mathrm{c})$, which contain the adverbial en fin de compte 'eventually/ ultimately' or the implicative verb finir par'end up', are much better, if not completely fine.

\footnotetext{
${ }^{4}$ See also Beavers (2012: 923) for a related observation.

${ }^{5}$ This version is only possible under the irrelevant reading where a killing event performed by the subject's referent takes place at the time specified by the time adverbial in the second clause, not identified with the action described in the first clause.

${ }^{6}$ The example (7) is adapted from a real occurrence found on https: / / tinyurl.com/y 7 geq $6 \mathrm{dh}$

${ }^{7}$ Lauer and Nadathur (2017) focus on the semantic differences between different subtypes of periphastic causatives, namely the English causatives make and cause, as well as the German causative lassen. The contrast obtained for French between $(8 \mathrm{a})$ and $(8 \mathrm{~b} / \mathrm{c})$ is very similar to what they observe for German lassen and English make.In particular, they claim that in the context (8), Das Erdbeben hat den Leuchtturm einstürzen lassen 'The earthquake made the tower collapse' is false, while Der starke Sturm hat den Leuchtturm einstürzen lassen 'The strong storm made the tower collapse' is true.
} 
(8) The lighthouse was built in a very sturdy foundation, designed to withstand high winds at the tower top, but the foundation sustained structural damage in an earthquake about ten years ago. Even that would have been fine, but this year, we had record-setting winds and the worst hurricane season anyone can remember, and given the prior damage, it could not take the extra strain provoked by the storms.

a. \#Le tremblement de terre a détruit le phare.

the earthquake has destroyed the lighthouse

'\#The earthquake destroyed the lighthouse.'

b. En fin de compte, ce tremblement de terre a détruit le phare! ultimately this earthquake has destroyed the lighthouse

'Ultimately, this earthquake destroyed the lighthouse!'

c. Et ce tremblement de terre a fini par détruire le phare! and this earthquake has finished by destroy the lighthouse

'And this earthquake eventually destroyed the lighthouse!'

Similarly, the sentence in (9) is generally judged more acceptable in the Wild West scenario (2a) than Katz's original sentence (2b) (or its French counterpart). ${ }^{8}$

(9) En fin de compte, l'armurier a fini par tuer le shériff. at the end of the day the gunsmith has finished by kille the sheriff 'At the end of the day, the gunsmith ended up killing the sheriff!'

A final new relevant observation is that the indirect reading of lexical causatives is also facilitated in contexts such that the (change of) state described by the verb is taken for granted-through, for instance, clefting - while what is under issue is the responsibility of the subject's referent, and/or what the ultimate causing event is. For instance, (10a) takes the lighthouse's destruction for granted through the clefting and the focusing of the subject, and is much better in the lighthouse scenario (8). Similarly, (10b) is also more acceptable in the Wild West scenario (2a); see also the attested French example (11). ${ }^{9}$

(10) a. C'est le [tremblement de terre $]_{F}$ qui a détruit le phare! it is the earthquake that has destroyed the lighthouse

'It is the earthquake that destroyed the lighthouse!'

b. It is the [gunsmith ('s mistake) $]_{F}$ that killed the sheriff!

\footnotetext{
${ }^{8}$ Interestingly, many of the examples through which Neeleman and Van de Koot (2012) argues for the felicity of the indirect reading of lexical causative verbs also contain the adverbial eventually.

${ }^{9}$ The example (11) is taken from an interview with Luz, a caricaturist of Charlie Hebdo, see https: / / tinyurl. $\mathrm{com} / \mathrm{y} 8$ eptebu
} 
(11) On a fait deux ou trois une sur Mahomet en quarante ans d'histoire We have made two or three front page on Mahomet in fourty years of history de Charlie. C'est ça qui a été mis en avant par les médias, et c'est ça of Charlie this is this that has been put to the fore by the media and this is this qui a tué nos amis.

that has killed our friends

'We made a couple of front pages on Mahomet in fourty years of Charlie's history, and this is what has been put to the fore by the media, and this is what killed our friends.'

Wolff (2003) famously proposed an alternative definition of direct causation, satisfied not only when there are no intermediate entities between the causer and the final causee, but also, if any intermediate entities are present, when those can be construed as an enabling condition rather than an intervening causer (that is, 'does something that is concordant with the tendency of the causer'). However, the direct causation constraint is not respected in the examples discussed above even under this redefinition of directness. For example, (9) or (10b) are acceptable in the Wild West scenario (2a) although the intermediate causer cannot be conceived as 'an enabling condition', as illustrated by the inappropriateness of the paraphrase in (12a) in the relevant context; also, (12b) is not an adequate paraphrase of (11). ${ }^{10}$

(12) a. Fred enabled the gunsmith to kill the sheriff.

b. The terrorists enabled the front pages to kill our friends.

To summarize, the causal relation expressed by lexical causative verbs may take place not only between two temporally adjacent eventualities, but also between two eventualities separated by intermediate events, even when intermediate causers are not enabling conditions in Wolff's sense. The indirect reading of lexical causative verbs is favoured by the adverbials en fin de compte 'ultimately, at the end of the day', the verb finir par 'end up, manage to' as well as contexts where the occurrence of the state reported by the lexical causative is taken for granted (through, e.g., the clefting of the subject). Explaining the facilitating effect of these constructions on the indirect reading is the topic of section 3. Moreover, Fodor's constraint on separate adverbial modification is relaxed with subject-denoting events, which I account for in section 4.

\section{Time in probabilistic causation}

\subsection{Introduction}

Let us compare again the following two sentences in the context of Katz's Wild West story:

(13) a. Fred killed the sheriff in the bar (by shooting him).

b. (By his negligence), the gunsmith killed the sheriff.

\footnotetext{
${ }^{10}$ The possibility to paraphrase $x V$-ed $y$ by $z$ enabled $x$ to $V y, z$ being the intermediate causer, is the main independent criterium provided by Wolff (2003) to check whether the intermediate entity can be conceived as an enabling condition.
} 
Let the variable $c$ represent the causing event, and $c_{S}$ the event the subject's referent participates in (the shooting in (13a), the repair of the gun in (13b)). The causing event $c$ can either be identical to $c_{S}$, or include $c_{S}$ as a proper part if there is an intermediate event $c_{I}$ between $c_{S}$ and the outcome $o$. In the latter case, $c=c_{S} \oplus c_{I}$.

Let us assume a context such in both $(13 \mathrm{a} / \mathrm{b})$, the constraint of temporal adjacency between $c_{S}$ and $o$ is violated. For instance, an intermediate event intervenes between $c_{S}$ (the act of Fred or the gunsmith) and the sheriff's death $o$ (a lethal internal bleeding the day after the gunshot in (13a), a gunfight in a bar in (13b)). Still, in such a context, the causal relation expressed in (13a) feels more direct than the one expressed in (13b), and I suspect that subjects would be more willing to accept the lexical causative in (13a) than in (13b) when temporal adjacency is not satisfied. How, then, should directness be defined, if not by temporal adjacency?

An obvious difference between (13a) and (13b) has to do with the causal impact of the event involving the subject $c_{S}$. In (13a), $c_{S}$ - the shooting - can be easily conceived as a sufficient cause for the sheriff's lethal bleeding and his ensuing death. In (13b), however, $c_{S}$ (the repair) is certainly not sufficient for the sheriff's death in the given scenario. A second related difference has to do with the way $c_{S}$ 'indicates' the sheriff's death if we restrict knowledge to the facts that pertain up to $t^{\prime}$, the right temporal boundary of $c_{S} \cdot{ }^{11}$ On one hand, Fred's shooting of the sheriff objectively points towards the sheriff's death, in that it raises the objective chance that the sheriff will die in the epistemic context up to $t$ '. Let us say that when $c_{S}$ raises the probability of $o$ in the epistemic context up to $t$ ', $c_{S}$ 'ab-initio causes' $o$. On the other hand, the gunsmith's repair, which is not known to be faulty at the time up to $t$ ', does not raise the probability of the sheriff's death at the time of the repair-it rather points to the contrary. That is, the gun's repair does not ab-initio cause the sheriff's death. It is only from a retrospective perspective, without limitations of knowledge of facts up to $t$ ', once the intermediate history between the gun's repair and the sheriff's death is taken into account (and the gunsmith's mistake identified), that the causal role of the gunsmith can be assessed. Let us say that in that case, $c_{S}$ 'ex-post-facto causes' the outcome $o$.

Ab-initio vs. ex-post-facto causality and the related statements will be defined more precisely below in the framework of Kvart's (2001) probabilistic account of singular causation. This ultimately will enable us to define causal directness independently from temporal adjacency, and to account for the data presented in the introduction.

\subsection{Kvart's theory of causation and the evolution of probabilities in time}

'Probabilistic causation' refers to a family of theories defining the relationship between cause and effect with the tools of probability theory. Central to these theories is the idea that causes change the probabilities of their effects, and more particularly that the occurrence of a cause increases

\footnotetext{
${ }^{11}$ See Varasdi (2014) on the notion of indicative properties. Indicativity will here be defined through probabilities, but the notion is not very different from Varasdi's notion of indicativity.
} 
the probability of the effect. Particularly interesting for us are the theories of causation between event particulars that depend on the way probabilities change over time, such at the theory of Kvart $(2001,2004) .^{12}$

Assume that the propositions $C_{S}$ and $O$ are descriptions of the events $c_{S}$ and $o$ respectively, and let be $W_{C_{S}}$ the world history just before $c_{S}$ occurs. ${ }^{13}$ How should probability increase be defined to capture the notion of cause? As Kvart observes, the most natural idea is to interpret probability increase as in (14). That is, given the world history up to $C_{S}$, the probability of $O$ given $C_{S}$ is higher than the probability of $O$ given $\neg C_{S}$.

$$
P\left(O \mid C_{S} \cdot W_{C_{S}}\right)>P\left(O \mid \neg C_{S} \cdot W_{C_{S}}\right)
$$

(ab-initio probability increase)

One of Kvart's crucial claims is that (14) is not an appropriate analysis of $C_{S}$ being a cause of $O$, despite the fact that this assumption is made in probabilistic theory such as Lewis's. ${ }^{14}$ The reason is that (14) is a function of the world up to $C_{S}$, but not at all a function of $W_{C_{S}, O}$, the intermediate history from $C_{S}$ to $O$ (which justifies his label ab-initio probability increase for the condition (14)). In other words, from $C_{S}, O$, and $W_{C_{S}}$, (14) fixes whether $C_{S}$ is a cause of $O$, 'regardless of what else transpires between' $C_{S}$ and $O$. And as Kvart forcefully argues, this does not do justice to the fact that whether $C_{S}$ is a cause of $O$ very much depends on what happens within the intermediate history. Kvart (2004) therefore proposes an alternative definition of probability increase to capture the notion of cause that takes the intermediate history between $C_{S}$ and $O$ into account. He calls this notion ex-post-facto probability increase, which is 'a sort of hindsight probability increase, from a bird's eye view, with the intermediate history unfolded' (Kvart 2004: 394). I illustrate the difference between ex-post-facto and ab-initio probability increase through three cases.

Case 1. Ex-post-facto probability increase can be easily illustrated through cases of ab-initio probability decrease (with ' $<$ ' instead of ' $>$ ' in (14)). Ex-post-facto probability increase despite of ab-initio probability decrease obtains when there is an intermediate event $c_{I}$ which increases the probability of $o$ when added to both sides of the ab-initio probability decrease condition, see (15a). Kvart calls such an intermediate event $c_{I}$ an increaser.

a. $\quad P\left(O \mid C_{S} \cdot C_{I} \cdot W_{C_{S}}\right)>P\left(O \mid \neg C_{S} \cdot C_{I} \cdot W_{C_{S}}\right)$

(ex-post-facto probability increase)

b. Sasha's bet improved her financial position.

\footnotetext{
${ }^{12}$ See also Eells (1991), and Hitchcock (2010) for an introduction to probabilistic causation in general and singular causation and the evolution of probabilities in time in particular, on which I partly rely in the presentation.

${ }^{13}$ The probability function $P$ takes propositions as its arguments, but 'events' is the formal term for these arguments in probability theory. In the case of singular causation, these 'events' correspond to what event semanticists call events (or to facts for some other authors). But since the formalism requires to make use of negation, disjunction or conjunction on these relata, these must be propositional entities. I assume that the (upper case) variables $C$ and $O$ correspond to propositions that are descriptions of the events picked up by the corresponding (lower cases) variables.

${ }^{14}$ See Kvart $(2001,2004)$ for detailed criticisms of Lewis's analysis of cause. The longer term project I do not have the space to pursue here is to offer a definition of Davidson's predicate cause used in section $\S 4$ in terms of Kvart's approach.
} 
Kvart illustrates ex-post-facto probability increase despite ab-initio probability decrease through an example similar to (15b) in a context such as the following. The Comeback Team had been weak for a long time, with few chances of improving during the next months. Nevertheless, Sascha bets a large portion of her financial worth on its winning $\left(c_{S}\right)$. Later but before the games start, a wealthy Hungarian start-upper bought the team, and acquired first-rate Belgian players. As a result, the team's performance was the best ever in the season $\left(\mathrm{c}_{I}\right)$. Sascha won her bet, and $o$ occurred-she improved her financial position. In this scenario, at the time of $c_{S}, c_{S}$ yielded a probability decrease of $o$ (since betting a lot of money on a weak team amounts to a waste of money). But given $c_{I}, c_{S}$ ultimately yielded a higher chance of $o$.

Case 2. Suppose now that ab-initio probability increase condition (14) obtains. For instance, in our previous lighthouse scenario (8), $c_{S}$ (the earthquake) is a probability increaser for $o$ (the lighthouse's destruction). Kvart's proposal is that in order to check whether $c_{S}$ is a cause of $o$, we need to check in the intermediate history between $c_{S}$ and $o$ if there is an intermediate event $c_{I}$ such that, if taken into account in the condition on both sides, reverses the inequality in (14), as in (16):

$$
P\left(O \mid C_{S} \cdot C_{I} \cdot W_{C_{S}}\right)<P\left(O \mid \neg C_{S} \cdot C_{I} \cdot W_{C_{S}}\right)
$$

If there is no such $c_{I}$ (that is, if the condition (16) does not obtain for any intermediate event), then ex-post-facto probability increase obtains, and $c_{S}$ is a stable increaser; $c_{S}$ can then be a cause of $o .{ }^{15}$ In the lighthouse scenario (8), there is indeed no such decreaser. The event $c_{S}$ can therefore be a cause of $o$, which corresponds to the intuition. ${ }^{16}$

Recall that the lexical causative statement (8a) is not felicitous in the lighthouse scenario. This confirms previous observations that the fact that $c_{S}$ is a cause of $o$ does not suffice to make the lexical causative acceptable in a default context (i.e. in absence of adverbials like ultimately, etc., see section 1). I will argue below that $c_{S}$ must be a sufficient ab-initio cause of $o$ for the lexical causative to be acceptable in a default context.

Case 3. Suppose again that (14) obtains but that there is a neutralizer intermediate event $c_{I}$, i.e. an event for which the condition (17) obtains:

$$
P\left(O \mid C_{S} \cdot C_{I} \cdot W_{C_{S}}\right)=P\left(O \mid \neg C_{S} \cdot C_{I} \cdot W_{C_{S}}\right)
$$

Imagine for instance that after the earthquake $\left(c_{S}\right)$, the city attributes funding to Mary because of the lighthouse's historical and artistic value. The lighthouse is fully and extensively renovated $\left(c_{I}\right)$. (Nevertheless, Mary's former husband intentionally burned it down for the insurance money and

\footnotetext{
${ }^{15}$ Cf. Kvart's (2001) THESIS 2: If (14) obtains, and (16) does not obtain for any intermediate event, then the requisite ex-post-facto probability increase obtains, and thus $c_{S}$ is a cause of $o$ (numbers and variables mine).

${ }^{16}$ As Kvart emphasizes, the existence of a stable increaser is a necessary but not sufficient condition for something's being a cause (see in particular Kvart 2004: section 3). $c_{S}$ must also be causally relevant to $o$. This happens if there is no intermediate event that neutralizes the potential causal relevance of $c_{S}$ to $o$. See Case 3 below for the definition of neutralizers.
} 
it gets completely destroyed $(o)$.) In this variant of the lighthouse scenario, the full renovation $c_{I}$ 'screens off' $c_{S}$ from $o$; that is, the earthquake $c_{S}$ loses its ab-initio causal impact on $o$ ex-postfacto. The event $c_{S}$ is not a cause of $o$, despite being an ab-initio probability increaser for $o$. This, again, corresponds to the intuition. Similarly, imagine in the lighthouse scenario that the storms in themselves were strong enough to destroy the lighthouse. Again, the earthquake loses its ab-initio causal impact on $o$ ex-post-facto, and is not a cause for $o$.

In sum, for $c_{S}$ to be a cause of $o$, it must have a stable increaser, and there should be no neutralizing intermediate event. Kvart $(2001,2004)$ discusses a number of additional cases to which I cannot do justice here. But I hope that this brief presentation showed how causal relations between events depend on the way in which probabilities evolve with time, and that the notion of 'some ab-initio positive causal impact' has to be distinguished from the notion of 'overall ex-post-facto positive causal impact'.

In the course of the linguistic analysis, I firstly argue that lexical causative statements by default trigger the implicature that $c_{S}$ is a sufficient ab-initio cause of $o$ given the world history $W_{C_{S}}$ up to $c_{S}$. In other words, they by default imply that it is already clear from the ab initio perspective that $c_{S}$ suffices to trigger the outcome $o$. Secondly, I claim that the constructions which increase the acceptability of the indirect use of lexical causatives, like en fin de compte/finalement 'ultimately, eventually' or finir par 'end up, manage to', or the clefting of the subject, all cancel this default inference (i.e., indicate that $c_{S}$ is not a sufficient ab-initio cause of $o$ ), and convey that $c_{S}$ 's causal impact is considered from a retrospective —ex-post-facto-perspective, once the intermediate history between $c_{S}$ and $o$ is taken into account. ${ }^{17}$

\subsection{The inference of directness of lexical causative verbs}

Let me now outline the main ingredients of the analysis. Firstly, I propose to redefine the linguistically relevant notion of causal directness through ab-initio sufficiency. ${ }^{18}$ That is, causal directness is satisfied when (18) below obtains. The condition (18) is satisfied if, given the world history $W_{C_{S}}$ immediately before $c_{S}$, the probability of $O$ given $C_{S}=1$ at $t^{\prime}$, the right temporal boundary of $c_{S} .{ }^{19}$

\footnotetext{
${ }^{17}$ The difference between the ab-initio and ex-post-facto causal impact of the event involving the subject's referent is also crucial to account for the fact that the so-called 'zero-change of state' reading of causative predicates is easier to obtain with agentive than with non-agentive subjects (see Martin 2015 and references therein): agentive subjects help to adopt the ab-initio perspective, while non-agentive ones often impose the ex-post-facto perspective.

${ }^{18}$ Lauer (2010: 21) already suggests that the inference of directness of lexical causatives (that he analyses as an implication rather than an implicature) may result from the fact that these predicates express causal sufficiency as well as causal necessity (as he puts it, 'a cause that is both necessary and sufficient cannot be very far from its effect'). Here, I do not commit to the view that lexical causatives express causal necessity.

${ }^{19}$ Note the condition (18) can be satisfied although $c_{S}$ is not a cause of $o$. This is where scenarios of causal overdetermination or preemption are relevant. For instance, Mandel (2003) ran an experiment involving a story in which the protagonist is first lethally poisoned, but then intentionally killed in a car accident, before the poison could yield its certain outcome. In this scenario, the administration of the poison ab-initio increases the probability of death to 1 , but is not judged a cause of the death by the subjects tested. Therefore, causal directness is not reduced to the condition (18). Rather, (18) defines what has to be the case for $c_{S}$ to be a direct cause of $o$, while it is independently established
} 


$$
P\left(O \mid C_{S} \cdot W_{C_{S}}\right)=1
$$

(ab-initio probability increase to 1 )

Secondly, I propose that in absence of information to the contrary, lexical causative statements trigger a defeasible inference (rather than an entailment) that causal directness (18) is satisfied. That is, in a default context, lexical causative statements require the event involving the subject's referent $c_{S}$ to be a sufficient ab-initio cause for $o$, regardless of what happens between $c_{S}$ and $o$ (this is the ab-initio requirement). Without entering into the details, I assume that this inference is obtained via a Gricean reasoning through the competition of lexical causatives with the corresponding periphrastic causatives (e.g. cause/make), which I suspect to strongly imply, and perhaps presuppose, that directness as I propose to redefine it through (18) is not satisfied, since they often involve an intervening causer with a causal contribution to $o$ not automatically triggered by $c_{S} .{ }^{20}$

The inference of directness as defined through (18) accounts for previous observations on the distribution of lexical causatives. Firstly, it accounts for the recurrent claim that lexical causatives involve something like physical manipulation of the object's referent by the subject's referent $S$. For the condition (18) is more likely to be satified when $S$ physically acts on the object's referent than when the object's referent is a full agent that $S$ incites to act: in the former case, but not the latter, $c_{S}$ can be conceived as a sufficient cause for $o$ in the epistemic context up to $t$ '. Take for instance the sharp contrast in (19), due to Ruwet (1972: 139).

(19) a. Delphine a fait entrer/a entré la voiture dans la garage.

'Delphine made enter/entered the car in the garage.'

b. Delphine a fait entrer/\# a entré les invités dans le salon.

'Delphine made/let enter/\#entered the guests in the living room.'

Given the world history $W_{C_{S}}$ immediately before $c_{S}$, it is easy to conceive Delphine's action on/with the car as a sufficient cause for the car's change of location, while it is odd to conceive Delphine's incentive to enter as a sufficient cause for the guest's change of location. This accounts for the fact that the lexical causative is felicitous in (19a) only (and note that the lexical causative in (19b) gets fine if the guests are in wheelchairs that they cannot drive themselves).

Secondly, the same condition (18) is also more easily satisfied if $c_{S}$ and $o$ are temporally adjacent or partly overlapping, for then, that $o$ obtains given that $c_{S}$ obtains can more easily be assessed regardless of the intermediate history, since the intermediate history is by definition empty in this case. This may explain the recurrent claim that lexical causatives require something like temporal adjacency between cause and effect.

that $c_{S}$ is a cause of $o$. This, as Kvart argues, requires ex-post-facto probability increase, which is not obtained in presence of a neutralizer such as the car accident in the scenario above.

${ }^{20}$ I owe to J.-P. Koenig the suggestion that directness is implied rather than entailed by lexical causatives. The competition between lexical and periphrastic causatives (addressed e.g. in Benz 2006) is out of the scope of this paper. A standardly overlooked difference between lexical causative statements and faire/make-statements is that with an agentive subject, the latter are not implicative; e.g. J'ai fait lire ton papier par les étudiants 'I made the students read your paper' does not entail (but rather strongly implies) that the students read your paper, while La curiosité a fait lire ton papier par les étudiants 'Curiosity made the students read your paper' does. 
However, the condition (18) can also be satisfied when $c_{S}$ and $o$ are temporally disjoint. Lee Oswald shot John Kennedy on November 221963 at 12.30, and Kennedy died at 13.00 the same day. But a bullet reached and crossed Kennedy's brain during the gunfire; his death was therefore certain before it actually took place (ignoring major violations of the laws of nature).

Thirdly, (18) is more easily satisfied with an intentional than with an accidental agent (such as the gunsmith in Katz's original scenario), because, as Copley (2018: fn. 5) underlines, causation associated with intention is robust: an intentional agent can adapt to changes in the environment in order for his chain of actions to be a sufficient cause for the intended outcome $o$. This contributes to explain Neeleman and Van de Koot's (2012) previous observation that lexical causatives are more acceptable in contexts where temporal adjacency is violated when the subject's referent is an intentional agent.

Fourthly, we expect subjects to vary in the way they judge lexical causatives to be acceptable under the indirect reading, since the same variation is observed with other types of true but pragmatically infelicitous statements (Noveck 2001 a.o). Subjects more sensitive to the inference of directness (18) are expected to be reluctant to endorse a lexical causative statement when (18) is not satisfied. For instance, the gun's repair by the gunsmith is not an ab-initio cause of the sheriff's death $o$ (i.e., (14) is not satisfied as the gun's repair is not a probability increser for $o$ up to $t^{\prime}$ ), and a fortiori not a sufficient ab-initio cause for it. Therefore, we expect the lexical causative statement ( $2 b)$ to be rejected by these speakers sensitive to the inference of directness. In the lighthouse scenario (8), the earthquake is an ab-initio cause of the lighthouse's destruction, but not a sufficient one (i.e., $\left.P\left(O \mid C_{S} \cdot W_{C_{S}}\right) \neq 1\right)$; we therefore also expect some speakers to reject (8b) in this scenario. Finally, Danlos's and Levin and Rappaport's examples (4)/(5) leave open the possibility that (18) is satisfied, since it may be that the shooting (or the poisoning) was an ab-initio-sufficient-cause of death. These examples are therefore expected not to raise a difficulty.

Fifthly, we also expect lexical causatives to be judged inappropriate by subjects more sensitive to the implicature in a context making clear that $c_{S}$ cannot raise the probability of $o$ to 1 regardless of what happens in the intermediate history between $c_{S}$ and $o$. This is the case in the example (20a).

(20) a. Paul killed Ana \#by forcing Sue to shoot her.

(inspired from Jackendoff 1972)

b. \#La presse lui a donné le prix Nobel.

the press her has given the prize Nobel

'\#The press coverage gave her the nobel Prize.'

In a default context, the by-clause in this example strongly suggests that Paul's action was not sufficient for Ana's death $o$; Sue also contributed to $o$ in a crucial way. ${ }^{21}$ Similarly, (20b) is weird, because the press coverage is not easily conceived as a sufficient cause for a Nobel Prize's attribution to an author.

\footnotetext{
${ }^{21}$ And note that in a context such that Paul physically forces Sue to shoot Ana, so that $c_{S}$ is more likely to be a sufficient ab-initio cause for $o$, the acceptability of (20a) increases.
} 


\subsection{Cancelling the inference of directness}

The inference of directness triggered by lexical causatives is, however, cancellable. I argue below that the linguistic constructions that facilitate the use of lexical causatives in the indirect readingthe adverbials en fin de compte/ au bout du compte 'ultimately, eventually', the implicative verb finir par 'end up, manage to' - do so because they indicate that (18) is not fulfilled. ${ }^{22}$ That is, these elements all convey that $c_{S}$ is not a sufficient ab-initio cause for $o$ in the epistemic context up to $t$ ' (the right temporal boundary of $c_{S}$ ), and that the causal impact of $c_{S}$ on $o$ is established from a retrospective perpective only, while the intermediate history between $c_{S}$ and $o$ is taken into account. I will call these constructions markers of delayed causation. Since, by assumption, the violation of the directness inference is the reason why lexical causatives are unacceptable in indirect causation contexts, the problem vanishes when this inference normally associated with lexical causatives is not triggered or is cancelled. Hence the fact that markers of delayed causation make the indirect reading of lexical causatives acceptable.

Let us first look more closely at causative statements of the form 'en fin de compte/ au bout $d u$ compte $P$ / finir par $P$ ' 'ultimately $\mathrm{P}$, eventually $\mathrm{P}$ ' (insightfully paraphrased as $P$ after a series of other things are taken into consideration by the Merriam Webster dictionary). In such statements, markers of delayed causation may have high or low scope, i.e. have either the whole causal chain on their scope, including the event involving the subject's referent, see (21a), or the causation event only, see (21b). When they help to license the indirect reading of lexical causatives, markers of delayed causation have their low scope reading.

(21) The executioner ultimately killed the prisoner.

a. After a series of events the executioner performed his job.

(high scope)

b. After a series of events the executioner's job caused the prisoner's death. (low scope)

I argue that these markers contribute in two crucial ways to the lexical causative statement that contains them. Firstly, such lexical causative statements imply that $c_{S}$ with an intermediate event $c_{i}$ are together jointly sufficient for $o$, see (22a). For instance, (22b) implies that the operation together with an intermediate event (e.g. subsequent complications) cause the dog's death. ${ }^{23}$

\footnotetext{
${ }^{22}$ Lauer and Nadathur (2017: \$3.2) relatedly propose that adverbials such as ultimately can shift what they call the evaluation time of periphrastic causative statements. They propose that this time is by default the time of the cause, which may correspond to the proposal made here that lexical causative statements are by default interpreted as ab-initio causal statements.

${ }^{23}$ This inference does not seem to be part of the assertive content of finir par $P$ 'ultimately P', for denials do not seem to be able to target it, as suggested by the infelicity of the following dialogue:
}

i. A. This operation ultimately killed the dog. ii. \#B. It's not true; it killed the dog right away-this vet is a true butcher! 
(22) a. $P\left(O \mid C_{S} \cdot C_{I} \cdot W_{C_{S}}\right)=1$

b. Cette opération a fini par tuer le chien.

(1st inference) this operation has finished by kill the dog

'The operation ultimately killed the dog.'

Secondly and relatedly, such lexical causative statements imply that $c_{S}$ is not a sufficient ab-initio cause for $o$, cf. (23). Statements of this type are compatible with situations where $c_{S}$ is either an ab-initio probability decreaser for $o$ (cf. e.g. the default interpretation of (22b)), or an ab-initio probability increaser for $o$ (although to a degree strictly inferior to 1), or neither of the two (i.e. when $\left.P\left(O \mid C_{S} \cdot W_{C_{S}}\right)=P\left(O \mid \neg C_{S} \cdot W_{C_{S}}\right)\right)$.

$$
P\left(O \mid C_{S} \cdot W_{C_{S}}\right)<1
$$

(2d inference)

Given their contributions (22a) and (23), markers of delayed causation are infelicitous when the action of the subject's referent is clearly a sufficient ab-initio cause for the outcome $o$, see (24). ${ }^{24}$

(24) a. The executioner beheaded the prisoner. He (\#ultimately/\#eventually) killed him!

b. John pressed the button on the automatic door. He (\#ultimately/\#eventually) opened it!

Markers of delayed causation are not the only way to neutralize the inference of causal directness, however. This inference, which is problematic for the indirect reading, is not triggered in the first place in a context where the causal role of intermediate events is already presupposed. Remember for instance the example (11) repeated below.

(11) We made a couple of front pages on Mahomet in fourty years of Charlie's history, and this is what has been put to the fore by the media, and this is what killed our friends.

In the context of the lexical causative statement (11) (taken from an interview with the caricaturist of Charlie Hebdo), the attack of January 72015 is taken for granted, as well as all other putative causes of the killing of Charlie Hebdo's team, and the killing event itself via the clefting of the subject. It is therefore from the beginning clear that the front page is not a sufficient ab-initio cause of $o$. Similarly, compare (20b) with its variant (25) below:

(25) C'est vrai, il a écrit un bon livre et le jury lui était très favorable. it is true he has written a good book and the committee him was very in favour

Mais en fin de compte, c'est la presse qui lui a donné le prix Nobel.

but at the end of the day it is the press that him has given the prize Nobel

'True, he wrote a good book and the committee was very in his favour. But at the end of the day, it is the press coverage that gave him the Nobel Prize.'

killed the dog ultimately), a position where the adverbial conveys an information which is central to the point made by the utterance. I leave open the question of how the inference of joint sufficiency should be analysed in such lexical causative statements.

${ }^{24}$ On that respect, markers of delayed causation share striking similarities with manage to $P$ as described by Baglini and Francez (2016), as reflected by the oddity of manage to open in the same contexts (see their ex. (27)). 
The example (25) is much more acceptable than (20b) because in the context of the lexical causative statement of (25), it is presupposed that the press coverage is not a sufficient cause for $o$. Again, the problematic inference of directness is neutralized. More generally, the clefting of the subject systematically facilitates the indirect reading because it presupposes the occurrence of the outcome $o$, and suggests that other causes of $o$ have been identified by making alternatives salient in discourse. This indicates that the causal relation is considered from a bird's eye view, with the intermediate history between $c_{S}$ and $o$ unrolled, rather than from an $a b$-initio point of view.

Finally, when the subject of the lexical causative refers to an event, the event description within the subject may also contribute to defeat the inference of directness. For instance in (3b), the event description 'the gunsmith's negligence' suggests by itself that all what happens between the gunsmith's repair and the sheriff's death is known in the context of the causative statement. It is therefore again clear from the start that $c_{S}$ is not an ab-initio cause for $o$. Therefore, the inference of directness is cancelled (or not triggered in the first place). ${ }^{25}$

\section{The constraint on separate adverbial modification}

Let us now turn to the questions of when and why separate adverbial modification is possible. I argue that we have to empirically distinguish between two different cases, namely, (i) separate modification of an event $e$ involving the subject's referent (e.g. a shooting) and an event $e^{\prime}$ causing a result state of the type encoded by the predicate (e.g. a killing event in the case of kill), such that $e$ causes $e^{\prime}$, and (ii) separate modification of a causing event $e^{\prime}$ (e.g. a killing event) and the ensuing caused state $s$ (e.g. a state of being dead).

\subsection{Separate modification of shooting events and killing events}

I take the sentences in (6) repeated below to illustrate that separate modification of the first subtype is possible with eventuality-denoting subjects, but not with entity-denoting subjects.

(6) a. Fred accidentally shot ${ }_{i}$ his dog on December 23! \#He eventually killed ${ }_{i}$ him on Dec. 25.

b. Fred accidentally shot his dog on December 23! This gunshot eventually killed him on December 25.

The eventuality predicate kill Fido is analysed as the bi-eventive predicate (26a), following, e.g., Schäfer (2008). We do not want to account for the unacceptability of (6a) by assuming that the causal relation between the shooting and the killing can only have temporally adjacent eventualities as relata, since we just argued at length in previous sections that cause can relate temporally distant eventualities. Rather, the problem of (6a) is a direct consequence of the fact that the adverbial must

\footnotetext{
${ }^{25}$ Note that with individual-denoting subjects too, the ex-post-facto perspective can be adopted through another element of the context, such as a by-phrase (cf. e.g. (13b)). Therefore, although event-denoting subjects in principle help to make the indirect reading felicitous, they are not necessary for this reading to obtain.
} 
scope on the causing event introduced by the lexical causative verb. The (standard) denotation of the adverbial on December 25 in (6) given in (26b) ensures this, see (26c), which gives the result of the composition of (26b) with (26a). ${ }^{26}$

(26) a. $\quad$ kill Fido $\leadsto \lambda e . \exists s($ cause $(e, s) \wedge \operatorname{dead}(s) \wedge$ theme $(s$, fido $))$

b. $\quad$ on December $25 \sim \lambda P \lambda e . P(e) \wedge \tau(e) \subseteq$ dec. 25

c. on December 25[kill Fido] $\sim[\lambda P \lambda e . P(e) \wedge \tau(e) \subseteq$ dec. 25]

$(\lambda e . \exists s(\operatorname{cause}(e, s) \wedge \operatorname{dead}(s) \wedge$ theme $(s$, fido $))=$

$\lambda e . \exists s(\operatorname{cause}(e, s) \wedge \operatorname{dead}(s) \wedge$ theme $(s$, fido $) \wedge \tau(e) \subseteq \operatorname{dec} .25)$

With an entity-denoting subject, the verbal predicate (26c) is combined with a Voice head (Kratzer 1996) that introduces an external argument $x$ of an event $e$, and such that $x$ is the agent of $e$, see (27a). (And note that $x$ may either act intentionally, or be an accidental agent, as in (9)). Applying (27a) to (26c), we obtain the verbal predicate (27b).

a. Voice $_{a g} \leadsto \lambda P \lambda x \lambda e$.agent $(e, x) \wedge P(e)$

b. Voice $a g$ [on December 25[kill Fido] $\leadsto$

$[\lambda P \lambda x \lambda$ e.agent $(e, x) \wedge P(e)]$

$(\lambda e . \exists s($ cause $(e, s) \wedge \operatorname{dead}(s) \wedge$ theme $(s$, fido $) \wedge \tau(e) \subseteq$ dec. 25$)=$

$\lambda x \lambda e . \exists s(\operatorname{agent}(e, x) \wedge \operatorname{cause}(e, s) \wedge \operatorname{dead}(s) \wedge$ theme $(s$, fido $) \wedge \tau(e) \subseteq$ dec. 25)

This obviously accounts for why sentence (6a) is contradictory: given that (27b) requires $x$ to perform on December 25 an event causing a state of being dead, there is no room left to identify this causing event with a previous action of $x$ taking place on December 23.

But then, what happens in (6b)? Pylkkänen (2008) assumes that event-denoting subjects are introduced by another Voice head, that identifies the event introduced by the subject $e$ (e.g., the gunshot in (6b)) and the causing event introduced by the verb (e.g., the killing event in (6b)). If such a head was involved in the semantic composition of $(6 \mathrm{~b})$, this sentence should be contradictory, given that the gunshot would have to take place both on December 23 and December 25. We therefore need another functional element than Pylkkänen's (2008) Voice. This head, that I will call Cause, is in charge of introducing an external argument $v$ that is an event or a state, ${ }^{27}$ and a causing relation between $v$ and the causing event $e$ introduced by the verbal predicate the head combines with, see (28a). Applying (28a) to (26c), we obtain the verbal predicate (28b), involving three different eventualities (and two causal relations).

$$
\begin{array}{ll}
\text { a. } & \text { Cause } \sim \lambda P \lambda v \lambda \text { e.event }(v) \vee \text { state }(v) \wedge \text { cause }(v, e) \wedge P(e) \\
\text { b. } \quad \text { Cause }[\text { on December } 25[\text { kill Fido]] } \sim \\
\\
{[\lambda P \lambda v \lambda \text { e.event }(v) \vee \text { state }(v) \wedge \text { cause }(v, e) \wedge P(e)]}
\end{array}
$$

\footnotetext{
${ }^{26}$ I assume that the adverbial on December 25 provides the Reichenbachian reference time, and that the bare accomplishment infinitive carries a perfective feature, responsible for the inclusion relation in (26b).

${ }^{27}$ In some cases as (3a), it seems that the causing eventuality denoted by the subject may be a state, which justifies the decision to leave the nature of the eventuality introduced by the subject unspecified.
} 


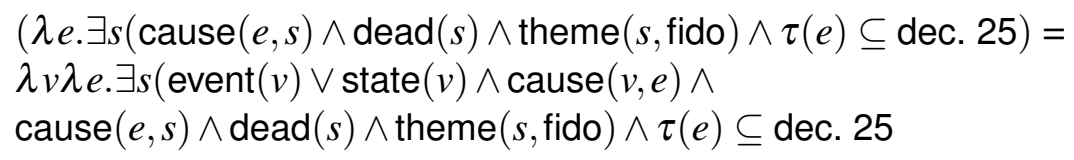

Let us now apply the predicate in (28b) to the definite event description $\imath v$.gunshot $(v)$, and derive the predicate in $(29 \mathrm{a})$, where the alternative that $v$ is a state is eliminated:

(29) a. The gunshot[Cause[On December 25[kill Fido]]] $\leadsto$

$\lambda e . \exists s(\operatorname{cause}(\imath v \cdot g u n s h o t(v), e) \wedge \operatorname{event}(v) \vee s \mid$ tate $e(y) \wedge \operatorname{cause}(e, s) \wedge$

$\operatorname{dead}(s) \wedge$ theme $(s$, fido $) \wedge \tau(e) \subseteq$ dec. 25)

b. Le coup de poignard d'hier a fini par le tuer ce matin.

the stabbing of yesterday has finished by him kill this morning

'Yesterday's stabbing eventually killed him this morning.'

We can now understand why sentence (6b) is acceptable. Given that the eventuality $v$ denoted by the subject causes the killing event $e$ denoted by the verb (rather than being identified with it), $v$ may, of course, take place before the event $e$ that must take place on December 25, e.g. on December 23. And observe that it is possible to add a temporal modifier within the subject DP that refers to a time different from the modifier applying to the VP, see (29b).

\subsection{Separate modification of killing events and caused states of being dead}

So far, we thus have accounted for the contrast between (6a) and (6b). The careful reader, however, will have noted that our representation of kill Fido on December 25 in (26c) leaves open the possibility that the caused state of being dead $s$ occurs after the time interval defined by the adverbial on December 25. For $s$ is not in the scope of this adverbial, and by assumption, cause can relate temporally distant events. Therefore, $(26 \mathrm{c})$ predicts that a causative lexical statement such as Fred killed Fido on December 25 can be true in situations where Fido dies after December 25. At this point, I am unsure whether this result is unwelcome or not. The oddity of the example (30a), which slightly modifies (4), suggests that it is. But (30b) is accepted by some speakers I consulted, which points to the possibility that the oddity of (30a) is not of a semantic nature. Also, one finds natural examples such as (30c), locating a killing event in the past, and death in the future.

(30) a. Fred killed Masha on Sunday. \#She (ultimately) died on Monday.

b. Lee Oswald killed President Kennedy on November 221963 at 12.30. He shot him as Kennedy rode in a motorcade through Dealey Plaza in downtown Dallas. Kennedy died at 13.00 at Parkland Memorial Hospital, where he was rushed after the shooting.

c. Already killed, but not dead yet.

If the examples in (30) turn out to be semantically acceptable despite some pragmatic anomalies for (30a), we can stick with (26c). Now, if examples in (30) turn out to be semantically anomalous 
because they violate the requirement that the causing event and the result state be in the scope of the temporal adverbial, we have to revise our semantics for kill in order to capture this requirement. One possibility suggested to me by C. Piñón (p.c.) is to include the caused state of being dead in the denotation of kill, and analyze kill Fido (on December 25) as in (31a-c). ${ }^{28}$

(31) a. kill Fido $\sim \lambda v \cdot \exists e \exists s(v=(e \oplus s) \wedge \operatorname{cause}(e, s) \wedge \operatorname{dead}(s) \wedge$ theme $(s$, fido $))$

b. $\quad$ on December $25 \leadsto \lambda P \lambda v \cdot P(v) \wedge \tau(v) \subseteq$ dec. 25

c. on December 25[kill Fido] $\sim[\lambda P \lambda v \cdot P(v) \wedge \tau(v) \subseteq$ dec. 25] $(\lambda v \cdot \exists e \exists s(v=(e \oplus s) \wedge$ cause $(e, s) \wedge \operatorname{dead}(s) \wedge$ theme $(s$, fido $))=$ $\lambda v . \exists e \exists s(v=(e \oplus s) \wedge \operatorname{cause}(e, s) \wedge \operatorname{dead}(s) \wedge$ theme $(s$, fido $) \wedge \tau(v) \subseteq \operatorname{dec} .25)$

This predicts examples such as (30) to be contradictory (and we can still account for (6a) vs. (6b) as before, via the Voice alternation). The price is that the sum $(e \oplus s)$ is not an eventuality in the usual sense. However, (31) captures the intuition that kill Fido denotes events and states.

\subsection{A final note on causative psych-verbs}

An intriguing property of causative psych-verbs is that they differ from non-psych verbs in that they allow for separate adverbial modification even with entity-denoting subjects, see (6a) vs. (32).

(32) Masha ${ }_{i}$ 's speech $_{j}$ on Monk's music on December 23 was quite something. And today $\mathbf{s h e}_{i} / \mathbf{i t}_{j}$ gave me the idea I needed for my term paper on phonotactic patterns! (uttered on Dec 25)

What is remarkable about (32) is that it is possible to identify Masha's speech on December 23 as the single one of her actions causing me to get the idea I needed for my paper (on December 25), and this even in presence of an individual-denoting subject. I claim that this specificity of psychverbs is due to the fact that their individual-denoting subjects may be reinterpreted as covert event descriptions. Technically, this translates in the view that with these verbs, subjects like Masha may either be introduced by the Voice head (27a), or by the Cause head (28a).

\section{References}

Baglini, R. and I. Francez (2016). The implications of managing. Journal of Semantics 33(3), 541-560.

Beavers, J. (2012). Resultative constructions. In R. Binnick (Ed.), The Oxford Handbook of Tense and Aspect, pp. 908-933. Oxford: Oxford University Press.

Benz, A. (2006). Partial blocking and associative learning. Linguistics and Philosophy 29(5), 587-615.

Copley, B. (2018). Dispositional causation. Glossa.

\footnotetext{
${ }^{28}$ See Rothstein 2004: 35 for an analysis of another subclass of accomplishments in terms of event sums (Rothstein, however, does not address standard causative predicates such as kill). A second possibility would be to analyze kill Fido in the general spirit of Piñón (2011), i.e. as predicates of event pairs $\langle e, s\rangle$ (i.e. analyse kill Fido as follows: $\lambda\langle e, s\rangle$.cause $(e, s) \wedge \operatorname{dead}(s) \wedge$ theme $(s$, fido $))$.
} 
Danlos, L. (2001). Event coreference in causal discourses. In F. Busa and P. Bouillon (Eds.), The Language of Word Meaning, pp. 216-242. Cambridge: Cambridge University Press.

Eells, E. (1991). Probabilistic Causality. Cambridge: Cambridge University Press.

Fodor, J. (1970). Three reasons for not deriving kill from cause to die. Linguistic Inquiry 1(4), 429-438.

Hitchcock, C. (2010). Probabilistic causation. Entry for the Stanford Encyclopedia of Philosophy. Jackendoff, R. S. (1972). Semantic Interpretation in Generative Grammar. Cambridge: MIT Press.

Katz, J. (1970). Interpretative semantics vs. generative semantics. Foundations of language 6/2, 220-259.

Kratzer, A. (1996). Severing the External Argument from its Verb. In J. Rooryck and L. Zaring (Eds.), Phrase Structure and the Lexicon. Dordrecht: Kluwer.

Kvart, I. (2001). The counterfactual analysis of cause. Synthese 127(3), 389-427.

Kvart, I. (2004). Causation: Probabilistic and counterfactual analyses. In J. Collins, N. Hall, and L. Paul (Eds.), Causation and counterfactuals, pp. 359-387. Cambridge: The MIT Press.

Lauer, S. (2010). Periphrastic causative verbs in english: What do they mean? The expression of causal necessity and causal sufficiency in ordinary english. Manuscript, Stanford University.

Lauer, S. and P. Nadathur (2017). Causal necessity, causal sufficiency, and the implications of causative verbs. Manuscript under review, University of Konstanz and Stanford University.

Levin, B. and M. Rappaport Hovav (1999). Two structures for compositionally derived events. In Proceedings of Semantics and Linguistic Theory (SALT) 9, pp. 199-223.

Mandel, D. (2003). Judgment dissociation theory: An analysis of differences in causal, counterfactual and covariational reasoning. Journal of Experimental Psychology 132(3), 419-434.

Martin, F. (2015). Explaining the link between agentivity and non-culminating causation. In Proceedings of Semantics and Linguistics Theory (SALT) 25, pp. 246-266.

Neeleman, A. and H. Van de Koot (2012). The linguistic expression of causation. In M. Everaert, M. Marelj, and T. Siloni (Eds.), The Theta System: Argument Structure at the Interface, pp. 20-51. Oxford University Press.

Noveck, I. A. (2001). When children are more logical than adults: Experimental investigations of scalar implicature. Cognition 78(2), 165-188.

Piñón, C. (2011). Event structure in event semantics. Talk at Chronos 10, Birmingham.

Pylkkänen, L. (2008). Introducing Arguments. Cambridge: MIT Press.

Rappaport Hovav, M. and B. Levin (2001). An event structure account of English resultatives. Language 77(4), 766-797.

Rothstein, S. (2004). Structuring Events. Malden/ Oxford: Blackwell Publishing.

Ruwet, N. (1972). Théorie syntaxique et syntaxe du français. Paris: Seuil.

Schäfer, F. (2008). The Syntax of (Anti-)Causatives. Amsterdam/Philadelphia: John Benjamins.

Varasdi, K. (2014). Making progressives: Necessary conditions are sufficient. Journal of Semantics 31, 179-207.

Wolff, P. (2003). Direct causation in the linguistic coding and individuation of causal events. Cognition 88(1), 1-48. 